\title{
28 Research Square \\ Proteomic Analysis of the Protective Effect of Early Heat Exposure Against Chronic Heat Stress in Broilers
}

\section{Darae Kang}

Animal biotechnology

Kwan Seob Shim ( $\nabla$ ksshim@jbnu.ac.kr)

Department of Animal Biotechnology, Jeonbuk National University, Jeonju 54896, Korea https://orcid.org/00000002-4996-3700

\section{Research}

Keywords: broiler, proteomics, heat stress, early heat exposure

Posted Date: August 26th, 2020

DOI: https://doi.org/10.21203/rs.3.rs-64761/v1

License: @ (1) This work is licensed under a Creative Commons Attribution 4.0 International License. Read Full License 


\section{Abstract}

\section{Background}

The increasing trend of global warming has affected the livestock industry through the heat stress caused to the animals. Among them, poultry are the most susceptible to heat stress, which results in serious production problems. Therefore, a better understanding of the mechanisms related to the thermal reactions and thermal resistance in poultry would be helpful toward resolving the production issues. In this study, whole proteome analysis was carried out to identify differentially expressed proteins in the liver tissue of broilers under chronic heat stress $\left(35^{\circ} \mathrm{C} / 24 \mathrm{~h}\right.$, each day between the broiler ages of $21-35$ days). Additionally, the effect of early heat exposure $\left(40^{\circ} \mathrm{C} / 24 \mathrm{~h}\right.$, for 1 day only on chicks at 5 days of age) was determined.

\section{Results}

In total, 277 differentially expressed proteins due to chronic heat stress were identified (132 downregulated and 145 upregulated). Of those, 95 proteins were regulated by early heat exposure (42 downregulated and 53 upregulated during chronic heat stress). Of the 95 proteins, 8 were related to actin metabolism. According to the KEGG analysis, the proteins were mainly involved in pathways for carbon metabolism and carbohydrate metabolism. Under chronic heat stress, the proteins involved in carbohydrate metabolism were expressed in such a way as to promote the metabolism of carbohydrates, which is the natural means to reduce body temperature but may well induce cell damage.

\section{Conclusion}

Early heat exposure reduced the heat stress-induced expression changes of select proteins, indicating the adaptability of the animal to chronic heat stress. The determination of the differentially expressed proteins in the liver proteome under chronic heat stress and early heat exposure suggests that the liver of broilers has various physiological mechanisms for regulating homeostasis to aid heat resistance.

\section{Background}

The poultry industry is influenced by a number of factors, including the climate, environment, breeding, specification conditions, and heat stress. Poultry are particularly sensitive to high temperatures because they lack the biological properties to release body heat; for example, they have no sweat glands and are covered with feathers (1). Heat stress leads consistently to reductions in feed consumption, feed efficiency, growth rate, and reproduction, thereby severely affecting the health of animals and impairing their immune functions (2). It can cause the dysfunction of organs and death, consequently leading to economic losses. Studies on broilers under high temperature stress have observed that their feed intake and growth rate are decreased (1), their respiration rates are increased (3), and the weights of organs such as the thymus, spleen, and bursa of Fabricius are reduced (4). The published studies on heat stress in poultry have mainly focused on the behavior, productivity, and blood biochemistry levels of the animals but not on the thermal reactions and mechanisms of thermal resistance.

Some studies have reported on the effects of early heat stress on chicks. Early heat was applied at either the pre- or post-hatching stages or at both stages combined to determine the effects of these various treatments in order to investigate whether the adaptability of chicks to heat stress could be improved through their earlier experience of a high temperature. The heat treatment before hatching (16-18 days of incubation) and at both hatching stages 
combined (16-18 days of incubation and in 3-day-old chicks) did not induce heat resistance in the chicks at 42 days, whereas the post-hatching heat exposure (3-day-old chicks) contributed to heat resistance (5). Early heattreated birds exhibited significantly reduced mortality and improved feed efficiency (6), and responded similarly to birds adapted to heat stress (7). These results indicated the protective effects of early heat exposure against heat stress later in life.

The techniques for studying genomics and proteomics are developing rapidly. Proteomic studies of heat stress effects have been mainly carried out on a number of plants and on animals such as dairy cows, beef cattle, pigs, and chickens. Proteins related to nuclear factor erythroid 2-related factor 2 (Nrf2)-mediated oxidative stress were identified in dairy cows under summer heat stress, and several biomarkers (including mitogen-activated protein kinase kinase 1 (MAP2K1), glutathione Stransferase mu 1 (GSTM1), and stress-induced phosphoprotein 1 (STIP1)) were identified in adipose tissue in response to heat stress (8). In heat-stressed pigs, there was an increase in the expression of heat-shock proteins that protect cells by degrading denatured proteins; inflammatory cytokines, such as glucose-regulated protein 94 (GRP94) and Serpin family A member 3 (SERPINA3); and glutathione peroxidase and glutathione $S$-transferase, which are related to immune responses (9). Thus, numerous proteins and regulatory functions related to heat stress have been and are still being studied through proteomic analysis. Through studies of the whole proteome of various organs, the effects of heat stress can be confirmed at the overall metabolic level rather than at fragmented parts, making a detailed understanding of the heat effects possible.

In this study, we conducted a proteomic analysis of broiler liver tissue under chronic heat stress, with/without early heat exposure, to identify the effects of early heat exposure in poultry. We found that the proteins that were differentially expressed in response to chronic heat stress were intended to maintain homeostasis in response to high temperatures, and the proteins of which the chronic heat stress-induced expression changes were renormalized through the early heat exposure were considered to be less affected by heat. Our results suggest that proteins can be changed through early heat exposure to increase their resistance to chronic heat stress.

\section{Methods}

\section{Animals and heat exposure conditions}

In total, 114 Ross chickens (1 day old) were purchased from Dongwoo Hatchery (Iksan, Korea). The chicks were weighed and randomly grouped into 3 groups of 48 chicks each without significant weight differences. Each group was further divided into 12 chicks per pen to represent 4 test replications. All 1-day-old chicks were initially raised at $34^{\circ} \mathrm{C}$ and the temperature was reduced by $2{ }^{\circ} \mathrm{C}$ weekly until it reached $24^{\circ} \mathrm{C}$. The humidity was maintained at $57 \% \pm$ $3 \%$ throughout. The chicks had access to water and feed ad libitum. The following were the heat treatment groups: Group CC, raised at a suitable temperature without heat exposure; Group $\mathrm{CH}$, chronic heat exposure at $35^{\circ} \mathrm{C}$ for $24 \mathrm{~h}$ each day between the ages of 21 and 35 days; and Group $\mathrm{HH}$, early heat exposure at $40^{\circ} \mathrm{C}$ for $24 \mathrm{~h}$ at 5 days of age and chronic heat exposure at $35^{\circ} \mathrm{C}$ for $24 \mathrm{~h}$ each day between the ages of 21 and 35 days (Fig. 1). At the end of the experimental period, the chickens were sacrificed, and the liver tissues were extracted, frozen with liquid nitrogen, and stored at $-80^{\circ} \mathrm{C}$ until analysis.

\section{Protein Extraction And Digestion}

An equal amount of liver tissue from each chicken was lysed in $1 \mathrm{~mL}$ of $8 \mathrm{M}$ urea and protein inhibitor with vortex mixing for $30 \mathrm{~s}$. Then, the mixture was sonicated for $3 \mathrm{~min}$ in an ice bath to homogenize the tissue. The 
homogenized tissue was then centrifuged at 14,000 rpm for $10 \mathrm{~min}$, following which the supernatant was obtained for protein concentration measurement with the bicinchoninic acid assay. Then, $100 \mu \mathrm{g}$ of protein was subjected to in-solution digestion. Thereafter, $100 \mu \mathrm{g}$ of depleted protein in $100 \mathrm{mM}$ Tris buffer $(\mathrm{pH} 3.0)$ in a total volume of $30 \mu \mathrm{L}$ was first incubated with $6 \mathrm{M}$ urea and $20 \mathrm{mM}$ dithiothreitol at $56^{\circ} \mathrm{C}$ for $30 \mathrm{~min}$ and then alkylated with fresh $50 \mathrm{mM}$ iodoacetamide in $100 \mathrm{mM}$ Tris buffer $\left(\mathrm{pH} \mathrm{8.0)}\right.$ for $30 \mathrm{~min}$ in the dark at ambient temperature $\left(24^{\circ} \mathrm{C}\right)$. The reaction was quenched with $100 \mathrm{mM}$ Tris buffer $(\mathrm{pH}$ 8.0) and the protein was enzymatically digested overnight at $37^{\circ} \mathrm{C}$ in a trypsin/LysC mix (1:50, enzyme:substrate). The reaction was quenched with formic acid and the peptides were desalted on an Oasis HLB column (Waters Corporation, Milford, MA, USA). Finally, the peptides obtained were dried using a speed vac.

\section{Liquid Chromatography-tandem Mass Spectrometry Analysis And Data Analysis}

The peptides were separated by liquid chromatography (LC) on an EASY-nLC 1000 system (Thermo Fisher Scientific, Rockford, IL, USA) equipped with a C18 column ( $2 \mu \mathrm{m}$ particle size, $50 \mu \mathrm{m}$ ID $\times 15 \mathrm{~cm}$ length; Thermo Fisher Scientific), using mobile phase A ( $0.1 \%$ formic acid in water) and B (0.1\% formic acid in $100 \%$ acetonitrile) at a flow rate of $300 \mathrm{~nL} / \mathrm{min}$. The gradient profile was set as follows: $5-40 \% \mathrm{~B}$ in $45 \mathrm{~min}$ and $40-80 \% \mathrm{~B}$ in $2 \mathrm{~min}$. Mass spectrometry (MS) analysis was performed using a Q Exactive mass spectrometer (Thermo Fisher Scientific) with the spray voltage set at $2.3 \mathrm{kV}$. MS spectra were collected at a resolution of 70,000 at $\mathrm{m} / \mathrm{z} 200(350-2000 \mathrm{~m} / \mathrm{z}$ mass range), followed by data-dependent higher-energy collisional dissociation (HCD) MS/MS spectra (at a resolution of 17,500 and collision energy of $25 \%$ ) of the 20 most abundant ions. A dynamic exclusion time of $30 \mathrm{~s}$ was used.

For the identification of the chicken liver protein, the raw files were searched against the UniProt Chicken database using the Percolator node in Proteome Discoverer (v1.4). Oxidation was chosen as the dynamic modification and carbamidomethyl as the static modification. The parent ion mass error was set to $\pm 10 \mathrm{ppm}$ and the fragment ion mass error to $\pm 0.02 \mathrm{Da}$. Peptides with full tryptic cleavage specificity were searched, with two missed cleavages allowed. The search parameters used were a precursor tolerance of $10 \mathrm{ppm}$ and a fragment ion tolerance of $0.02 \mathrm{Da}$. Scaffold Q + S was used for the label-free quantitation of the proteins. All identified proteins were normalized by protein size and total spectrum count number, and the normalized data were filtered with a confidence level $(\mathrm{Cl})$ of $>95 \%$. For further filtering, the false discovery rate was set to 0.05 and the count number to $>0$ for 2 out of 2 replicates.

\section{Gene Ontology Enrichment Analysis}

The differentially expressed proteins were clustered using the Database for Annotation, Visualization and Integrated Discovery bioinformatics resources (DAVID v6.8, https://david.ncifcrf.gov/) with the Gene Ontology (GO) database, and the probability value was corrected with the Bonferroni method. The classifications from the Gallus gallus database were applied.

Pathway enrichment analysis was carried out using the WEB-based Gene SeT AnaLysis Toolkit (WebGestalt, http://www.webgestalt.org/) with the Kyoto Encyclopedia of Genes and Genomes (KEGG) database. The classifications were again from the Gallus gallus database. The probability value was corrected with the Benjamini-Hochberg method. 


\section{Validation Of Proteins By Their Gene Expression}

Total RNA was extracted from the liver tissue using an RNA extraction kit (Bioneer, Daejeon, Korea) according to the manufacturer's instructions. The RNA concentration and purity were measured using the $\mu$ Drop Plate (NanoDrop, Thermo Fisher Scientific). cDNA was synthesized from $1 \mu \mathrm{g}$ of total RNA using the AccuPower RocketScript Cycle RT PreMix (dT20) (Bioneer). The randomly selected gene primers were designed with Primer 3 software (v.0.4.0) and are shown in Supplemental Table S1. The reverse transcription quantitative polymerase chain reaction (RT-qPCR) was conducted using the SsoFast EvaGreen Supermix (Bio-Rad, Hercules, CA, USA) on a CFX96 real-time PCR detection system (Bio-Rad). The RT-qPCR thermal cycle conditions were as follows: $95^{\circ} \mathrm{C}$ for $5 \mathrm{~min}$, followed by 40 cycles of $95^{\circ} \mathrm{C}$ for 5 and $60^{\circ} \mathrm{C}$ for $30 \mathrm{~s}$. The relative gene expression levels were calculated with the $2^{-\triangle \triangle C t}$ method $\{$ Livak, 2001 \#131\} and normalized against the level of glyceraldehyde 3-phosphate dehydrogenase (GAPDH).

\section{Statistical analysis}

All experimental data were analyzed with the SAS 9.4 program and are expressed as the mean \pm SE. Differences were analyzed using analysis of variance, and statistical differences among groups were analyzed with Duncan's multiple-range test. Statistical significance was set at $P<0.05$.

\section{Results}

\section{Differentially expressed proteins in response to chronic heat stress and early heat exposure}

In total, 277 proteins (132 downregulated and 145 upregulated) were differentially expressed between the control and $\mathrm{CH}$ groups (Table S2). However, the chronic heat affect was reduced by early heat exposure for 95 (42 downregulated and 53 upregulated by chronic heat stress) of the 277 proteins (Table 1). The heat map and graph of the expression patterns of the differentially expressed proteins in the various groups are shown in Fig. $2.0 f$ the 95 proteins that were positively affected by the early heat exposure, the putative interferon-stimulated gene 12 (ISG12) protein was the most highly expressed in response to chronic heat stress. Induced by interferons, ISG12 enables a variety of biologically active states, such as protection against viral replication and increase of the host defenses through the activation of immune cells, major histocompatibility complex antigens, etc. Therefore, the role of this protein against viral infections and in regulating the immune system may be regulated by early heat exposure. 
Table 1

List of differentially expressed proteins of positive effected by early heat exposure

\begin{tabular}{|c|c|c|c|c|c|c|c|}
\hline \multirow[t]{2}{*}{ No } & \multirow[t]{2}{*}{ UniProt } & \multirow[t]{2}{*}{ Description } & \multirow[t]{2}{*}{ Name } & \multirow{2}{*}{$\begin{array}{l}\mathrm{MW} \\
(\mathrm{kDa})\end{array}$} & \multicolumn{3}{|c|}{ Score } \\
\hline & & & & & CC & $\mathrm{CH}$ & $\mathrm{HH}$ \\
\hline \multicolumn{8}{|c|}{ Low expressed by chronic heat stress } \\
\hline 1 & F1NJG4 & Cytochrome P450 CYP2D49 & CYP2D6 & 58 & 1 & 0.40 & 0.80 \\
\hline 2 & F1NBI2 & $\begin{array}{l}\text { Uroporphyrinogen_deCOase } \\
\text { domain-containing protein }\end{array}$ & UROD & 36 & 1 & 0.34 & 0.70 \\
\hline 3 & F1NK40 & Uncharacterized protein & A2ML4 & 163 & 1 & 0.09 & 1.18 \\
\hline 4 & P05094 & $\begin{array}{l}\text { Alpha-actinin-1 (Alpha-actinin } \\
\text { cytoskeletal isoform) (F-actin } \\
\text { cross-linking protein) (Non-muscle } \\
\text { alpha-actinin-1) }\end{array}$ & ACTN1 & 102 & 1 & 0.37 & 0.88 \\
\hline 5 & F1P298 & $\begin{array}{l}\text { Amidohydro-rel domain-containing } \\
\text { protein }\end{array}$ & AMDHD1 & 37 & 1 & 0.04 & 0.85 \\
\hline 6 & P28337 & $\begin{array}{l}\text { Aminomethyltransferase, } \\
\text { mitochondrial, EC 2.1.2.10 (Glycine } \\
\text { cleavage system T protein, GCVT) }\end{array}$ & AMT & 42 & 1 & 0.29 & 0.94 \\
\hline 7 & - & Calpain-11 & CAPN11 & 80 & 1 & 0.11 & 0.69 \\
\hline 8 & Q5ZHR7 & Clathrin light chain & CLTA & 24 & 1 & 0.21 & 0.83 \\
\hline 9 & A0A1L1RWF6 & Calponin & CNN3 & 37 & 1 & 0.25 & 0.71 \\
\hline 10 & A0A1D5PLS2 & Citrate synthase & CS & 60 & 1 & 0.34 & 0.93 \\
\hline 11 & A0A1L1RWI4 & Uncharacterized protein & CYP2AC2 & 57 & 1 & 0.08 & 0.68 \\
\hline 12 & A0A3Q2U335 & ADF-H domain-containing protein & DBNL & 45 & 1 & 0.41 & 0.98 \\
\hline 13 & P28675 & $\begin{array}{l}\text { Decorin (Bone proteoglycan II) (PG- } \\
\text { S2) }\end{array}$ & DCN & 40 & 1 & 0.26 & 1.06 \\
\hline 14 & A0A3Q3AA40 & $\begin{array}{l}\text { Eukaryotic translation initiation } \\
\text { factor } 3 \text { subunit G, elF3g } \\
\text { (Eukaryotic translation initiation } \\
\text { factor } 3 \text { RNA-binding subunit, elF-3 } \\
\text { RNA-binding subunit) (Eukaryotic } \\
\text { translation initiation factor } 3 \\
\text { subunit 4) }\end{array}$ & EIF3G & 29 & 1 & 0.33 & 0.90 \\
\hline 15 & A0A1D5P5T1 & $\begin{array}{l}\text { Eukaryotic translation initiation } \\
\text { factor } 3 \text { subunit K, elF3k } \\
\text { (Eukaryotic translation initiation } \\
\text { factor } 3 \text { subunit 12) (elF-3 p25) }\end{array}$ & EIF3K & 27 & 1 & 0.04 & 0.73 \\
\hline 16 & A0A3Q2TU97 & $\begin{array}{l}\text { Eukaryotic translation initiation } \\
\text { factor } 4 \mathrm{E}\end{array}$ & EIF4E & 25 & 1 & 0.22 & 1.16 \\
\hline 17 & A0A1D5PU09 & PH domain-containing protein & FERMT2 & 81 & 1 & 0.15 & 0.83 \\
\hline 18 & E1C6R4 & $\begin{array}{l}\text { Peptidylprolyl isomerase, EC } \\
\text { 5.2.1.8 }\end{array}$ & FKBP8 & 44 & 1 & 0.03 & 0.69 \\
\hline
\end{tabular}




\begin{tabular}{|c|c|c|c|c|c|c|c|}
\hline \multirow[t]{2}{*}{ No } & \multirow[t]{2}{*}{ UniProt } & \multirow[t]{2}{*}{ Description } & \multirow[t]{2}{*}{ Name } & \multirow{2}{*}{$\begin{array}{l}\text { MW } \\
(\mathrm{kDa})\end{array}$} & \multicolumn{3}{|c|}{ Score } \\
\hline & & & & & $\mathrm{CC}$ & $\mathrm{CH}$ & $\mathrm{HH}$ \\
\hline 19 & P21872 & $\begin{array}{l}\text { Trifunctional purine biosynthetic } \\
\text { protein adenosine-3 [Includes: } \\
\text { Phosphoribosylamine-glycine } \\
\text { ligase, EC 6.3.4.13 (Glycinamide } \\
\text { ribonucleotide synthetase, GARS) } \\
\text { (Phosphoribosylglycinamide } \\
\text { synthetase) }\end{array}$ & GART & 107 & 1 & 0.17 & 0.93 \\
\hline 20 & A0A1D5PZ32 & $\begin{array}{l}\text { GB1/RHD3-type G domain- } \\
\text { containing protein }\end{array}$ & GBP4L & 70 & 1 & 0.04 & 1.13 \\
\hline 21 & F1NLE4 & $\begin{array}{l}\text { Glutathione synthetase, GSH-S, EC } \\
\text { 6.3.2.3 }\end{array}$ & GSS & 52 & 1 & 0.07 & 0.68 \\
\hline 22 & P20057 & Hemopexin & HPX & 43 & 1 & 0.31 & 0.83 \\
\hline 23 & A0A1D5P1W7 & $\begin{array}{l}\text { Importin N-terminal domain- } \\
\text { containing protein }\end{array}$ & KPNB1 & 120 & 1 & 0.37 & 0.88 \\
\hline 24 & Q5ZK33 & $\begin{array}{l}\text { Mitochondrial proton/calcium } \\
\text { exchanger protein (Leucine zipper- } \\
\text { EF-hand-containing } \\
\text { transmembrane protein 1) }\end{array}$ & LETM1 & 86 & 1 & 0.06 & 0.71 \\
\hline 25 & A0A3Q3B025 & $\begin{array}{l}\text { LIM zinc-binding domain- } \\
\text { containing protein }\end{array}$ & LIMA1 & 85 & 1 & 0.20 & 0.56 \\
\hline 26 & A0A1D5PMD9 & $\begin{array}{l}\text { Carboxylic ester hydrolase, EC } \\
\text { 3.1.1.- }\end{array}$ & LOC769704 & 59 & 1 & 0.38 & 1.02 \\
\hline 27 & P00698 & $\begin{array}{l}\text { Lysozyme C, EC 3.2.1.17 (1,4-beta- } \\
\text { N-acetylmuramidase C) (Allergen } \\
\text { Gal d IV) (allergen Gal d 4) }\end{array}$ & LYZ & 16 & 1 & 0.21 & 0.86 \\
\hline 28 & P14105 & Myosin-9 & MYH9 & 227 & 1 & 0.21 & 0.46 \\
\hline 29 & F1NTJ5 & Myosin IB & MY01B & 125 & 1 & 0.26 & 0.73 \\
\hline 30 & A0A1L1RKT0 & $\begin{array}{l}\text { Glycylpeptide N- } \\
\text { tetradecanoyltransferase, EC } \\
\text { 2.3.1.97 }\end{array}$ & NMT1 & 57 & 1 & 0.04 & 0.34 \\
\hline 31 & 013154 & $\begin{array}{l}\text { Protein kinase } \mathrm{C} \text { and casein kinase } \\
\text { substrate in neurons protein } \\
2 \text { (Focal adhesion protein of } \\
52 \mathrm{kDa} \text {, FAP52) }\end{array}$ & PACSIN2 & 56 & 1 & 0.44 & 0.99 \\
\hline 32 & A0A1D5PLK6 & BR01 domain-containing protein & PDCD6IP & 97 & 1 & 0.26 & 0.72 \\
\hline 33 & Q5ZLX0 & $\begin{array}{l}\text { Cytochrome b5 heme-binding } \\
\text { domain-containing protein }\end{array}$ & PGRMC2 & 22 & 1 & 0.02 & 0.48 \\
\hline 34 & 042265 & $\begin{array}{l}\text { Proteasome subunit alpha type- } 1 \text {, } \\
\text { EC 3.4.25.1 (Macropain subunit } \\
\text { C2) (Multicatalytic endopeptidase } \\
\text { complex subunit C2) (Proteasome } \\
\text { component C2) }\end{array}$ & PSMA1 & 29 & 1 & 0.16 & 0.64 \\
\hline 35 & E1BWG7 & Phosphotriesterase related protein & PTER & 39 & 1 & 0.17 & 0.94 \\
\hline
\end{tabular}




\begin{tabular}{|c|c|c|c|c|c|c|c|}
\hline \multirow[t]{2}{*}{ No } & \multirow[t]{2}{*}{ UniProt } & \multirow[t]{2}{*}{ Description } & \multirow[t]{2}{*}{ Name } & \multirow{2}{*}{$\begin{array}{l}\mathrm{MW} \\
(\mathrm{kDa})\end{array}$} & \multicolumn{3}{|c|}{ Score } \\
\hline & & & & & $\mathrm{CC}$ & $\mathrm{CH}$ & $\mathrm{HH}$ \\
\hline 36 & A0A1D5PNR0 & RNA-binding protein $8 \mathrm{~A}$ & RBM8A & 19 & 1 & 0.02 & 0.94 \\
\hline 37 & A0A1D5PDV6 & Ribosomal protein S19 & RPS19 & 15 & 1 & 0.39 & 0.86 \\
\hline 38 & Q5ZM66 & 40 S ribosomal protein $\$ 26$ & RPS26 & 13 & 1 & 0.34 & 0.93 \\
\hline 39 & A0A1L1S0C5 & PX domain-containing protein & SNX1 & 58 & 1 & 0.04 & 0.73 \\
\hline 40 & P0DMQ6 & $\begin{array}{l}\text { Sorbitol dehydrogenase, SDH, EC } \\
\text { 1.1.1.- (Polyol dehydrogenase) }\end{array}$ & SORD & 38 & 1 & 0.34 & 1.17 \\
\hline 41 & A0A1L1RY95 & $\begin{array}{l}\text { UBIQUITIN_CONJUGAT_2 domain- } \\
\text { containing protein }\end{array}$ & UBE2N & 17 & 1 & 0.37 & 1.14 \\
\hline 42 & A0A1D5NZ55 & $\begin{array}{l}\text { UDP-glucose glycoprotein } \\
\text { glucosyltransferase } 1\end{array}$ & UGGT1 & 180 & 1 & 0.06 & 0.18 \\
\hline \multicolumn{8}{|c|}{ High expressed by chronic heat stress } \\
\hline 43 & F1NGM0 & Uncharacterized protein & EHD3 & 61 & 1 & 2.09 & 0.84 \\
\hline 44 & F1NNH9 & $\begin{array}{l}\text { TPR_REGION domain-containing } \\
\text { protein }\end{array}$ & TOMM70 & 67 & 1 & 3.34 & 0.90 \\
\hline 45 & Q5ZHT1 & $\begin{array}{l}\text { Acyl-CoA dehydrogenase family } \\
\text { member } 11\end{array}$ & ACAD11 & 87 & 1 & 8.29 & 1.16 \\
\hline 46 & F1NEF6 & Uncharacterized protein & ACAD 9 & 37 & 1 & 9.75 & 1.16 \\
\hline 47 & Q5ZKG5 & $\begin{array}{l}\text { Low molecular weight } \\
\text { phosphotyrosine protein } \\
\text { phosphatase }\end{array}$ & ACP1 & 18 & 1 & 20.07 & 1.16 \\
\hline 48 & E1BZT9 & Acetyl-coenzyme A synthetase & ACSS1L & 58 & 1 & 5.39 & 1.43 \\
\hline 49 & - & Uncharacterized protein & AKR1B10L4 & 36 & 1 & 10.32 & 1.16 \\
\hline 50 & P09572 & $\begin{array}{l}\text { Sodium/potassium-transporting } \\
\text { ATPase subunit alpha- } 1 \text {, } \\
\mathrm{Na}(+) / \mathrm{K}(+) \text { ATPase alpha- } 1 \text { subunit }\end{array}$ & ATP1A1 & 113 & 1 & 6.28 & 1.16 \\
\hline 51 & A0A1D5PBD2 & Uncharacterized protein & ATPIF1 & 13 & 1 & 4.32 & 1.43 \\
\hline 52 & A0A3Q3AZM2 & Uncharacterized protein & CALM1 & 16 & 1 & 23.16 & 1.16 \\
\hline 53 & A9CP13 & D-serine dehydratase & CHDSD & 40 & 1 & 34.27 & 1.16 \\
\hline 54 & Q91882 & Protein kinase $\mathrm{C}$ inhibitor & chPKCl & 14 & 1 & 3.93 & 1.33 \\
\hline 55 & A0A1D5PCT4 & Coronin & COR01C & 64 & 1 & 2.41 & 0.95 \\
\hline 56 & P55164 & Beta-crystallin A2 & CRYBA2 & 23 & 1 & 83.20 & 1.16 \\
\hline 57 & A0A1L1RKJ5 & Carboxypeptidase, EC 3.4.16.- & CTSA & 53 & 1 & 34.27 & 9.25 \\
\hline 58 & Q5F412 & Dynein light chain & DYL1 & 10 & 1 & 2.73 & 1.15 \\
\hline 59 & F1N9U8 & ETF domain-containing protein & ETFA & 34 & 1 & 19.93 & 1.16 \\
\hline
\end{tabular}




\begin{tabular}{|c|c|c|c|c|c|c|c|}
\hline \multirow[t]{2}{*}{ No } & \multirow[t]{2}{*}{ UniProt } & \multirow[t]{2}{*}{ Description } & \multirow[t]{2}{*}{ Name } & \multirow{2}{*}{$\begin{array}{l}\mathrm{MW} \\
(\mathrm{kDa})\end{array}$} & \multicolumn{3}{|c|}{ Score } \\
\hline & & & & & CC & $\mathrm{CH}$ & $\mathrm{HH}$ \\
\hline 60 & F1NHG6 & Uncharacterized protein & FAM162A & 17 & 1 & 85.75 & 1.16 \\
\hline 61 & A0A1D5P316 & $\begin{array}{l}\text { Peptidylprolyl isomerase, EC } \\
\text { 5.2.1.8 }\end{array}$ & FKBP3 & 26 & 1 & 26.39 & 1.16 \\
\hline 62 & A0A1D5NYG3 & Uncharacterized protein & FLNB & 284 & 1 & 2.03 & 0.96 \\
\hline 63 & P21872 & $\begin{array}{l}\text { Trifunctional purine biosynthetic } \\
\text { protein adenosine- } \\
3 \text { [Includes: Phosphoribosylamine- } \\
\text { glycine ligase }\end{array}$ & GART & 107 & 1 & 9.67 & 1.16 \\
\hline 64 & P00504 & $\begin{array}{l}\text { Aspartate aminotransferase, } \\
\text { cytoplasmic, cAspAT }\end{array}$ & GOT1 & 46 & 1 & 2.94 & 1.33 \\
\hline 65 & F1NIJ6 & Glucose-6-phosphate isomerase & GPI & 26 & 1 & 14.09 & 1.16 \\
\hline 66 & - & Uncharacterized protein & GSPT1 & 68 & 1 & 5.13 & 1.16 \\
\hline 67 & A0A1D5P338 & Glutathione reductase & GSR & 50 & 1 & 21.09 & 9.25 \\
\hline 68 & F1NQS2 & Uncharacterized protein & GSTAL3 & 26 & 1 & 13.86 & 1.16 \\
\hline 69 & E1BUB6 & Uncharacterized protein & GSTT1L & 28 & 1 & 4.29 & 1.45 \\
\hline 70 & E1BRU7 & Uncharacterized protein & HKDC1 & 102 & 1 & 2.08 & 0.94 \\
\hline 71 & P35915 & $\begin{array}{l}\text { Hydroxymethylglutaryl-CoA lyase, } \\
\text { mitochondrial }\end{array}$ & HMGCL & 34 & 1 & 10.26 & 1.16 \\
\hline 72 & Q5ZM98 & Stress-70 protein, mitochondrial & HSPA9 & 73 & 1 & 4.82 & 1.16 \\
\hline 73 & Q5ZKA2 & $\begin{array}{l}\text { Isoleucine-tRNA ligase, } \\
\text { mitochondrial }\end{array}$ & IARS & 147 & 1 & 2.92 & 1.36 \\
\hline 74 & E1BWB9 & Uncharacterized protein & INF2 & 144 & 1 & 4.96 & 1.16 \\
\hline 75 & Q6IEC5 & Putative ISG12(2) protein & ISG12(2) & 10 & 1 & 89.96 & 40.86 \\
\hline 76 & A0A1D5PAF0 & Uncharacterized protein & LOC107080643 & 27 & 1 & 4.67 & 1.05 \\
\hline 77 & E1BRI5 & $\begin{array}{l}\text { Abhydrolase_2 domain-containing } \\
\text { protein }\end{array}$ & LYPLA2 & 25 & 1 & 3.16 & 1.46 \\
\hline 78 & E1BTL0 & $\begin{array}{l}\text { Transket_pyr domain-containing } \\
\text { protein }\end{array}$ & OGDHL & 115 & 1 & 3.25 & 1.16 \\
\hline 79 & F1P0M2 & Uncharacterized protein & PCCA & 79 & 1 & 3.00 & 0.95 \\
\hline 80 & A0A1L1RW22 & $\begin{array}{l}\text { CTP_transf_like domain-containing } \\
\text { protein }\end{array}$ & РCYT2 & 40 & 1 & 18.41 & 1.16 \\
\hline 81 & Q5ZLT2 & Protein-serine/threonine kinase & PDK3 & 44 & 1 & 2.92 & 1.30 \\
\hline 82 & A0A1L1RXJ1 & Uncharacterized protein & QARS & 96 & 1 & 7.57 & 1.16 \\
\hline 83 & E1COF3 & Uncharacterized protein & RAB7A & 24 & 1 & 2.10 & 0.96 \\
\hline 84 & Q5ZM11 & Arginine-tRNA ligase, cytoplasmic & RARS & 75 & 1 & 2.08 & 0.94 \\
\hline
\end{tabular}




\begin{tabular}{|c|c|c|c|c|c|c|c|}
\hline \multirow[t]{2}{*}{ No } & \multirow[t]{2}{*}{ UniProt } & \multirow[t]{2}{*}{ Description } & \multirow[t]{2}{*}{ Name } & \multirow{2}{*}{$\begin{array}{l}\mathrm{MW} \\
(\mathrm{kDa})\end{array}$} & \multicolumn{3}{|c|}{ Score } \\
\hline & & & & & CC & $\mathrm{CH}$ & $\mathrm{HH}$ \\
\hline 85 & P41263 & Retinol-binding protein 4 & RBP4 & 23 & 1 & 16.20 & 1.16 \\
\hline 86 & E1BSA7 & Uncharacterized protein & SEC24A & 120 & 1 & 5.85 & 1.16 \\
\hline 87 & A0A1D5NVD 4 & Uncharacterized protein & SRSF7 & 28 & 1 & 13.35 & 1.16 \\
\hline 88 & A0A1D6UPQ3 & $\begin{array}{l}\text { AA_TRNA_LIGASE_II domain- } \\
\text { containing protein }\end{array}$ & TARS & 91 & 1 & 6.43 & 1.43 \\
\hline 89 & A0A1L1RMM0 & Uncharacterized protein & tсp-1 & 58 & 1 & 11.99 & 1.16 \\
\hline 90 & F1NU71 & $\begin{array}{l}\text { Mitochondrial import inner } \\
\text { membrane translocase subunit } \\
\text { TIM44 }\end{array}$ & TIMM44 & 51 & 1 & 4.23 & 1.42 \\
\hline 91 & P08629 & Thioredoxin, Trx & TXN & 12 & 1 & 3.43 & 1.70 \\
\hline 92 & A0A1D5PWT4 & $\begin{array}{l}\text { Thioredoxin domain-containing } \\
\text { protein }\end{array}$ & TXN2 & 16 & 1 & 64.63 & 1.16 \\
\hline 93 & - & Uncharacterized protein & UGT2A1 & 61 & 1 & 24.35 & 8.10 \\
\hline 94 & A0A1D5PEU7 & $\begin{array}{l}\text { CN hydrolase domain-containing } \\
\text { protein }\end{array}$ & VNN1 & 57 & 1 & 6.66 & 1.16 \\
\hline 95 & 093277 & WD repeat-containing protein 1 & WDR1 & 67 & 1 & 10.56 & 1.16 \\
\hline
\end{tabular}

To further compare the differences among the $\mathrm{CC}, \mathrm{CH}$, and $\mathrm{HH}$ groups, the functions of the differentially expressed proteins were categorized using the WebGestalt toolkit and the GO resource, in which the three main functional categories are biological process, cellular component, and molecular function. Using the 95 proteins that were positively affected by early heat treatment, we obtained the top $10 \mathrm{GO}$ terms for the three main categories (Table S3 and Fig. 3). In the biological process category, genes were associated with the small molecule metabolic process (13 genes), actin filament-based process ( 7 genes), actin cytoskeleton organization ( 7 genes), and cofactor metabolic process ( 7 genes). Additionally, the most related term was glutathione metabolic process. In the cellular component category, actomyosin had the highest significance among the $\mathrm{GO}$ terms, with the 4 genes actinin alpha 1 (ACTN1), fermitin family homolog 2 (FERMT2), LIM domain and actin binding 1 (LIMA1), and filamin B (FLNB) being associated with the actomyosin, stress fiber, contractile actin filament bundle, actin filament bundle, focal adhesion, cell-substrate adherens junction, adherens junction, and actin cytoskeleton terms. In the molecular function category, the genes were strongly associated with actin filament binding, with the 5 genes ACTN1, FERMT2, LIMA1, myosin heavy chain 9 (MYH9), and myosin 1B (MYO1B) being enriched in the actin filament binding, actin binding, cytoskeletal protein binding, and protein-containing complex binding terms.

On the basis of the KEGG pathway analysis with the WebGestalt toolkit, we identified 13 significant pathways among the $\mathrm{CC}, \mathrm{CH}$, and $\mathrm{HH}$ groups (Table 2). Many pathways were involved in carbohydrate metabolism, including those of glycolysis/gluconeogenesis, citrate cycle, pentose and glucoronate interconversions, fructose and mannose metabolism, starch and sucrose metabolism, glyoxylate and dicarboxylate metabolism, and propanoate metabolism. 
List of KEGG pathways

\begin{tabular}{|c|c|c|c|c|}
\hline Term ID & Term & No. & Genes & p-value \\
\hline \multicolumn{5}{|c|}{ Carbohydrate metabolism } \\
\hline 00010 & Glycolysis / Gluconeogenesis & 3 & ACSS1L, GPI, HKDC1 & 0.02 \\
\hline 00020 & Citrate cycle (TCA cycle) & 2 & CS, OGDHL & 0.04 \\
\hline 00040 & Pentose and glucoronate interconversions & 2 & SORD, UGT2A1 & 0.02 \\
\hline 00051 & Fructose and mannose metabolism & 2 & HKDC1, SORD & 0.04 \\
\hline 00500 & Starch and sucrose metabolism & 3 & GPI, HKDC1, UGT2A1 & 0.01 \\
\hline 00630 & Glyoxylate and dicarboxylate metabolism & 4 & AMT, CS, PCCA, ACSS1L & 0.004 \\
\hline 00640 & Propanoate metabolism & 2 & ACSS1L, PCCA & 0.04 \\
\hline \multicolumn{5}{|c|}{ Metabolism of cofactors and vitamins } \\
\hline 00670 & One carbon pool by folate & 2 & AMT, GART & 0.01 \\
\hline 00860 & Porphyrin and chlorophyll metabolism & 2 & UGT2A1, UROD & 0.03 \\
\hline \multicolumn{5}{|c|}{ Xenobiotics biodegradation and metabolism } \\
\hline 00982 & Drug metabolism (cytochrome P450) & 2 & GSTAL3, GSTT1L & 0.04 \\
\hline 00983 & Drug metabolism (other enzymes) & 3 & LOC769704, GSTAL3, GSTT1L & 0.02 \\
\hline 00980 & Metabolism of xenobiotics by cytochrome P450 & 2 & GSTT1L, UGT2A1 & 0.05 \\
\hline \multicolumn{5}{|c|}{ Translation } \\
\hline 00970 & Aminoacyl-tRNA biosynthesis & 4 & IARS, QARS, RARS, TARS & 0.001 \\
\hline \multicolumn{5}{|c|}{ Amino acid metabolism } \\
\hline 00270 & Cysteine and methionine metabolism & 2 & GOT1, GSS & 0.06 \\
\hline \multicolumn{5}{|c|}{ Metabolism of other amino acids } \\
\hline 00480 & Glutathione metabolism & 3 & GSR, GSS, GSTT1L & 0.01 \\
\hline \multicolumn{5}{|c|}{ Lipid metabolism } \\
\hline 00140 & Steroid hormone biosynthesis & 2 & CYP2D6, UGT2A1 & 0.06 \\
\hline
\end{tabular}

\section{Gene Expression Analysis For Validation Of The Abundant Protein}

We analyzed the mRNA expression levels of glutamic-oxaloacetic transaminase 1 (GOT1), phosphoribosylglycinamide formyltransferase, phosphoribosylglycinamide synthetase, phosphoribosylaminoimidazole synthetase (GART), glutathione S-transferase theta 1-like (GSTT1L), 2-oxoglutarate dehydrogenase-like, mitochondrial (OGDHL), UDP glucuronosyltransferase family 2 member A1 (UGT2A1), arginyltRNA synthetase, cytoplasmic (RARS), cytochrome P450 2D6 (CYP2D6), isoleucyl-tRNA synthetase (IARS), and glutathione synthetase (GSS) (Fig. 4). The OGDHL and UGT2A1 expression levels were significantly higher in the $\mathrm{CH}$ 
and $\mathrm{HH}$ groups than in the $\mathrm{CC}$ group $(P<0.05)$, where the pattern was similar to that of their protein expression levels; that is, the highest in the $\mathrm{CH}$ group, lowest in the CC group, and moderate in the HH group. The GOT1, GART, GSTT1L, RARS, CYP2D6, and GSS expression levels were significantly higher in the $\mathrm{CH}$ group than in the $\mathrm{CC}$ and $\mathrm{HH}$ groups $(P<0.05)$, whereas IARS expression was significantly lower in the $\mathrm{CH}$ group $(P<0.05)$. GOT1, GART, and GSTT1L showed the same gene and protein expression patterns, whereas CYP2D6, IARS, and GSS showed the opposite expression patterns.

\section{Discussion}

Heat stress reduces the health and activity of animals as well as agricultural productivity. Although many research studies on the physiological reactions of animals to heat have been conducted, there are no direct countermeasures to offset the negative effects and any such measure is limited to general environmental temperature reduction and feed additives. The use of early heat exposure for reducing the thermal stress of broilers has emerged as an economically viable method compared with other countermeasures. Although strategies for reducing heat stress have increased the heat resistance of animals, the productivity of the animals in the hot summer season has yet to be fully normalized. Therefore, a detailed understanding of the biological responses to heat stress is necessary to aid the development of techniques for normalizing the heat-related physiological and metabolic responses of animals. Among the livestock affected by high temperatures, poultry was selected as it is the most susceptible to heat and therefore the most suitable for studying the metabolic response during heat exposure.

Given that protein metabolism in animals can be altered by heat, researchers have studied the heat-induced proteomic responses in various animals, such as cattle, pigs, and birds. For example, \{Victoria Sanz Fernandez, 2015 \#241\} reported that heat stress affects carbohydrate metabolism in pigs. Heat stress decreases ATP production through oxidative phosphorylation and increases energy production through aerobic sugar degradation, thereby significantly altering intracellular energy (11). This is similar to the Warburg effect that is used in tumor cells to gain energy (12). Our differentially expressed proteins were consistent with the results of these reports, in that proteins in pathways of carbohydrate metabolism-such as those in glycolysis/gluconeogenesis (acetyl-CoA synthetase 2-like, mitochondrial isoform X1 (ACSS1L), glucose 6-phosphate isomerase (GPI), hexokinase domaincontaining 1 (HKDC1)), the citrate cycle (OGDHL), starch and sucrose metabolism (GPI, HKDC1, UGT2A1), and propanoate metabolism (ACSS1L, propionyl-CoA carboxylase subunit alpha (PCCA)) -were upregulated in the liver tissue of the $\mathrm{CH}$ chickens compared with that of the $\mathrm{CC}$ and $\mathrm{HH}$ chickens. The overall metabolic process is shown in Fig. 5.

In glycolysis, HKDC1 (a member of the hexokinase family) plays an important role in regulating glucose metabolism by catalyzing the ATP-dependent phosphorylation of glucose-6-phosphate at the first step of the glycolytic pathway (13). Although the specific biological functions of HKDC1 are still unclear, it has been proposed to play an especially greater role in glucose metabolism when the fetus needs to be supplied with sufficient nutrients during pregnancy (14). In a study of the sugar degradation pathways of various hyperthermophilic archaea, the extremophiles were found to degrade glucose, maltose, cellobiose, and starch through modified Embden-Meyerhof pathways (15). Additionally, it has been found that enzyme activity increases exponentially at high temperatures (above $\left.55^{\circ} \mathrm{C}\right)(16)$. GPI, which catalyzes the reversible isomerization of glucose-6-phosphate and fructose-6-phosphate, plays a role in glycolysis and gluconeogenesis in the cytoplasm and is also involved in the pentose phosphate pathway (17). This protein has been studied as a neurotrophic factor for promoting the survival of skeletal and sensory neurons and inducing immunoglobulin secretion, and as a tumor-secreting cytokine that plays a role in tumor angiogenesis and metastasis and cell migration, proliferation, and apoptosis $(18,19)$. GPI deficiency induces hemolytic anemia, 
whereas its overexpression is related to carcinogenesis and its elevated serum level is used as a prognostic biomarker of colon, rectal, breast, lung, and kidney cancers (20). Therefore, the upregulation of both HKDC1 and GPI by chronic heat stress would promote glycolysis and activate pathways to obtain energy from glucose in the body. However, such overexpression may induce cell damage as well. Sorbitol dehydrogenase (SORD), which converts sorbitol to fructose in the polyol pathway, is closely related to various diabetic complications (viz., neuropathy, retinopathy, cataracts, and nephropathy) (21). The decreased expression of SORD causes an excessive accumulation of sorbitol, leading to osmotic damage to the retinal endothelial cells and pericytes through oxidativenitridation stress and activation of the protein kinase $\mathrm{C}$ pathways, with resultant inflammation and growth factor imbalance (22). Our results showed that the expression of SORD was reduced by chronic heat stress, which is coincident with the finding that SORD is inactivated with increasing temperature (23). However, the SORD reduction in the $\mathrm{CH}$ group was recovered in the $\mathrm{HH}$ group, indicating that early heat exposure plays a protective role against heat stress in the liver cells.

In the tricarboxylic acid (TCA) cycle, the main precursor acetyl-CoA is essential for energy generation toward the mitochondria (24). During a lack of carbohydrate intake or utilization, acetate is used as an essential source for producing acetyl-CoA under ketogenic conditions and is involved in heat generation (25). In broilers, ACSS1L catalyzes the synthesis of acetyl-CoA for use in glycolysis. The feed intake of the $\mathrm{CH}$ broilers was significantly decreased compared with that of the CC broilers, and thus the expression of ACSS1L was increased in that group owing to the insufficient nutrients for metabolism. Citrate synthase (CS) catalyzes the condensation reaction to form citrate from oxaloacetate and acetyl-CoA, which are the first steps in the TCA cycle. It is also used as an enzymatic marker of intact mitochondria (26). CS activity has been found to be decreased by mitochondrial dysfunction and inhibited by oxidative stress (27). Another study confirmed the protective function of CS in thermally stressed yeast cells (28), where deletion of the CS-coding gene cit/ resulted in temperature-sensitive ROS accumulation, nuclear and DNA fragmentation, and phosphatidylserine translocation, all of which are hallmarks of cytological apoptosis. From the results of our study, we can postulate that heat stress causes damage to the mitochondria as a result of the downregulation of CS, but early heat exposure can regulate the CS expression level. OGDHL, which is located in the mitochondria, is also a TCA cycle-related enzyme and indirectly responsible for the induction of apoptosis. The increased expression of OGDHL contributes to the acceleration of the TCA cycle (29). It was found in a previous study that OGDHL overexpression significantly induced ROS production and lipid peroxidation in the mitochondria of cervical cancer cells and resulted in apoptosis by inducing caspase-3 activity and PARP protein expression, whereas OGDHL suppression significantly inhibited these processes and promoted cell proliferation (30). These results suggest that overexpressed OGDHL plays an important role in inducing ROSmediated apoptosis. In our study, chronic heat stress induced OGDHL expression, which suggests that it may induce ROS production followed by cell damage. Thus, the increase in carbohydrate metabolism observed in chronically heat-stressed broilers may be the result of a lack of energy in the body.

In general, heat stress increases energy consumption for panting to dissipate heat, maintaining homeostasis, and protecting the cells (31). In a study on the fatty acid levels in broilers under heat stress, the plasma concentration of non-esterified fatty acids was reduced in the heat-stressed animals, and better absorption and storage of triglycerides in the intestine or liver were observed (32). This is because the heat emitted from fat metabolism is higher than that generated through carbohydrate metabolism. Similarly, in heat-stressed dairy cows, energy was preferentially supplied by the carbohydrates to reduce the body's own heat generation (33). However, in our study, chronic heat stress, which increases the requirement of a considerable amount of energy, increased the expression of enzymes of the acyl-CoA dehydrogenase (ACAD) family (i.e., ACAD11 and ACAD9) to obtain energy through fat. This confirms that a considerable amount of energy is required, along with a reduction in feed intake, in response to 
chronic heat stress. Although heat stress significantly reduced the nutrient (and thus potential energy) intake by the broilers, a large amount of energy was needed from metabolism to cope with the stress. Thus, the expression of carbohydrate metabolism-related proteins changed.

\section{Conclusions}

The feasibility of the early heat exposure method for reducing heat stress in broilers was studied, as this method has been reported to have a protective effect against heat stress. Although there are published studies on the effects of heat stress on cell growth, heat-shock protein expression, hormones, and blood parameters, to our best knowledge, no research has been conducted on the mechanisms related to the thermal reactions. Thus, in this study, we carried out a proteomic analysis to confirm which proteins/processes are regulated by early heat exposure under chronic heat stress. The results showed that chronic heat stress affected the expression of proteins involved in carbohydrate metabolism and carbon metabolism, which generally result in less heat production than lipid metabolism for energy production. If the expression of these proteins increases, cell damage may increase as well. However, the early heat treatment recovered the proteins that were induced (or reduced) by chronic heat stress, indicating that early heat exposure increases the heat resistance of the animal to inhibit biological damage. The knowledge about these physiological changes would be useful for the development of programs for breeding animals with high heat resistance. Moreover, this early heat exposure method and the results of our study may be helpful in reducing heat stress in animals, thereby improving animal welfare in general.

\section{List Of Abbreviations}

Nrf2: Nuclear factor erythroid 2-related factor 2; MAP2K1: Mitogen-activated protein kinase kinase 1; GSTM1: Glutathione S-transferase mu 1; STIP1: stress-induced phosphoprotein 1; GRP94: Glucose-regulated protein 94; SERPINA3: Serpin family A member 3; LC: liquid chromatography; GAPDH: Glyceraldehyde 3-phosphate dehydrogenase; ISG12: Interferon-stimulated gene 12; ACTN1: Actinin alpha 1; FERMT2: fermitin family homolog 2;

LIMA1: LIM domain and actin binding 1; FLNB: filamin B; MYH9: myosin heavy chain 9; MY01B: myosin 1B; GOT1: Glutamic-oxaloacetic transaminase 1; GART: Phosphoribosylgrlycinamide formyltransferase, phosphoribosylglycinamide synthetase, phosphoribosylaminoimidazole synthetase; GSTT1L: Glutathione Stransferase theta 1-like; OGDHL: 2-oxoglutarate dehydrogenase-like, mitochondrial; UGT2A1: UDP glucuronosyltransferase family 2 member A1; RARS: Arginyl-tRNA synthetase, cytoplasmic; CYP2D6: Cytochrome P450 2D6; IARS: Isoleucyl-tRNA synthetase; GSS: Glutathione synthetase; ACSS1L: Acetyl-CoA synthetase 2-like, mitochondrial isoform X1; GPI: Glucose 6-phosphate isomerase; HKDC1: Hexokinase domain-containing 1; PCCA: Propionyl-CoA carboxylase subunit alpha; SORD: Sorbitol dehydrogenase; TCA: tricarboxylic acid; CS: Citrate synthase; ACAD: Acyl-CoA dehydrogenase;

\section{Declarations}

\section{Ethics approval and consent to participate}

Experimental procedures for animals were approved by the Animal Ethics Committee of the Jeonbuk National University (CBNU2018-097), Republic of Korea.

\section{Consent for publication}

Not applicable. 
Availability of data and material

The datasets produced and/or analyzed during the current study are available from the corresponding author on reasonable request.

\section{Competing interests}

The authors declare that they have no competing interests.

\section{Funding}

This research was supported by the National Research Foundation of Korea (NRF) grant funded by the Korea government (MSIT) (No. 2020R1I1A3A04038058).

\section{Authors' contributions}

Writing-original draft preparation, DK; writing-review and editing, KS; conceiving and design, DK and KS; supervision, KS; analysis, DK; Both authors read and approved the final manuscript.

\section{Acknowledgements}

DK: Department of Animal Biotechnology, Jeonbuk National University, Korea, PhD.

KS: Department of Animal Biotehchnology, Jeonbuk National University, Korea, Professor.

\section{References}

1. Park S-O, Hwangbo J, Ryu C-M, Yoon J-S, Park B-S, Kang H-K, et al. Effects of extreme heat stress and continuous lighting on growth performance and blood lipid in broiler chickens. Journal of The Korean Oil Chemists Society. 2013;30.

2. Siegel H. Physiological stress in birds. Bioscience. 1980;30(8):529-34.

3. Richards S-A, editor Physiology of thermal panting in birds. Annales de Biologie Animale Biochimie Biophysique; 1970: EDP Sciences.

4. Quinteiro-Filho WM, Ribeiro A, Ferraz-de-Paula V, Pinheiro M, Sakai M, Sá LRMd, et al. Heat stress impairs performance parameters, induces intestinal injury, and decreases macrophage activity in broiler chickens. Poultry Science. 2010;89(9):1905-14.

5. Tona K, Onagbesan O, Bruggeman V, Collin A, Berri C, Duclos M, et al. Effects of heat conditioning at d 16 to 18 of incubation or during early broiler rearing on embryo physiology, posthatch growth performance and heat tolerance. Archiv für Geflügelkunde. 2008;72(2):75-83.

6. Yahav S, Hurwitz S. Induction of thermotolerance in male broiler chickens by temperature conditioning at an early age. Poultry Science. 1996;75(3):402-6.

7. Hassan AM, Reddy PG. Early age thermal conditioning improves broiler chick's response to acute heat stress at marketing age. American Journal of Animal and Veterinary Sciences. 2012;7(1):1-6.

8. Zachut M, Kra G, Livshitz L, Portnick Y, Yakoby S, Friedlander G, et al. Seasonal heat stress affects adipose tissue proteome toward enrichment of the Nrf2-mediated oxidative stress response in late-pregnant dairy cows. Journal of proteomics. 2017;158:52-61. 
9. Cui Y, Hao Y, Li J, Bao W, Li G, Gao Y, et al. Chronic heat stress induces immune response, oxidative stress response, and apoptosis of finishing pig liver: a proteomic approach. International journal of molecular sciences. 2016;17(5):393.

10. Victoria Sanz Fernandez M, Johnson JS, Abuajamieh M, Stoakes SK, Seibert JT, Cox L, et al. Effects of heat stress on carbohydrate and lipid metabolism in growing pigs. Physiological reports. 2015;3(2):e12315.

11. Verberk W, Sommer U, Davidson R, Viant M. Anaerobic metabolism at thermal extremes: a metabolomic test of the oxygen limitation hypothesis in an aquatic insect. Integrative and Comparative Biology. 2013;53(4):609-19.

12. Pavlides S, Whitaker-Menezes D, Castello-Cros R, Flomenberg N, Witkiewicz AK, Frank PG, et al. The reverse Warburg effect: aerobic glycolysis in cancer associated fibroblasts and the tumor stroma. Cell cycle. 2009;8(23):3984-4001.

13. Li G-H, Huang J-F. Inferring therapeutic targets from heterogeneous data: HKDC1 is a novel potential therapeutic target for cancer. Bioinformatics. 2013;30(6):748-52.

14. Guo C, Ludvik AE, Arlotto ME, Hayes MG, Armstrong LL, Scholtens DM, et al. Coordinated regulatory variation associated with gestational hyperglycaemia regulates expression of the novel hexokinase HKDC1. Nature communications. 2015;6:6069.

15. Stetter KO. Extremophiles and their adaptation to hot environments. FEBS letters. 1999;452(1-2):22-5.

16. Hansen T, Oehlmann M, Schönheit P. Novel type of glucose-6-phosphate isomerase in the hyperthermophilic archaeon Pyrococcus furiosus. Journal of bacteriology. 2001;183(11):3428-35.

17. Cordeiro A, Godoi P, Silva C, Garratt RC, Oliva G, Thiemann OH. Crystal structure of human phosphoglucose isomerase and analysis of the initial catalytic steps. Biochimica et Biophysica Acta (BBA)-Proteins and Proteomics. 2003;1645(2):117-22.

18. Ma Y-T, Xing X-F, Dong B, Cheng X-J, Guo T, Du H, et al. higher autocrine motility factor/glucose-6-phosphate isomerase expression is associated with tumorigenesis and poorer prognosis in gastric cancer. Cancer management and research. 2018;10:4969.

19. Watanabe H, Takehana K, Date M, Shinozaki T, Raz A. Tumor cell autocrine motility factor is the neuroleukin/phosphohexose isomerase polypeptide. Cancer research. 1996;56(13):2960-3.

20. Somarowthu S, Brodkin HR, D’Aquino JA, Ringe D, Ondrechen MJ, Beuning PJ. A tale of two isomerases: compact versus extended active sites in ketosteroid isomerase and phosphoglucose isomerase. Biochemistry. 2011;50(43):9283-95.

21. Ohtsuka Y, Yabunaka N, Watanabe I, Noro H, Fujisawa H, Agishi Y. Thermal stress and diabetic complications. International Journal of Biometeorology. 1995;38(2):57-9.

22. Obrosova IG. Increased sorbitol pathway activity generates oxidative stress in tissue sites for diabetic complications. Antioxidants \& redox signaling. 2005;7(11-12):1543-52.

23. Marini I, Moschini R, Del Corso A, Mura U. Complete protection by a-crystallin of lens sorbitol dehydrogenase undergoing thermal stress. Journal of Biological Chemistry. 2000;275(42):32559-65.

24. Akram M. Citric acid cycle and role of its intermediates in metabolism. Cell biochemistry and biophysics. 2014;68(3):475-8.

25. Arun V, Mino T, Matsuo T. Biological mechanism of acetate uptake mediated by carbohydrate consumption in excess phosphorus removal systems. Water Research. 1988;22(5):565-70.

26. Marco R, Pestaña A, Sebastian J, Sols A. Oxaloacetate metabolic crossroads in liver. Enzyme compartmentation and regulation of gluconeogenesis. Molecular and cellular biochemistry. 1974;3(1):53-70. 
27. Scaini G, Rochi N, Benedet J, Ferreira GK, Teodorak BP, Comim CM, et al. Inhibition of brain citrate synthase activity in an animal model of sepsis. Revista Brasileira de terapia intensiva. 2011;23(2):158-63.

28. Lee YJ, Hoe KL, Maeng PJ. Yeast cells lacking the CIT1-encoded mitochondrial citrate synthase are hypersusceptible to heat-or aging-induced apoptosis. Molecular biology of the cell. 2007;18(9):3556-67.

29. Fujisawa K, Terai S, Takami T, Yamamoto N, Yamasaki T, Matsumoto T, et al. Modulation of anti-cancer drug sensitivity through the regulation of mitochondrial activity by adenylate kinase 4 . Journal of Experimental \& Clinical Cancer Research. 2016;35(1):48.

30. Sen T, Sen N, Noordhuis MG, Ravi R, Wu TC, Ha PK, et al. OGDHL is a modifier of AKT-dependent signaling and NF-kB function. PloS one. 2012;7(11):e48770.

31. Zhou W, Fujita M, Yamamoto S. Thermoregulatory responses and blood viscosity in dehydrated heat-exposed broilers (Gallus domesticus). Journal of Thermal Biology. 1999;24(3):185-92.

32. Sands J, Smith M. Effects of dietary manganese proteinate or chromium picolinate supplementation on plasma insulin, glucagon, glucose and serum lipids in broiler chickens reared under thermoneutral or heat stress conditions. International Journal of Poultry Science. 2002;1(5):145-9.

33. Baumgard L, Wheelock J, Sanders S, Moore C, Green H, Waldron M, et al. Postabsorptive carbohydrate adaptations to heat stress and monensin supplementation in lactating Holstein cows. Journal of Dairy Science. 2011;94(11):5620-33.

\section{Figures}

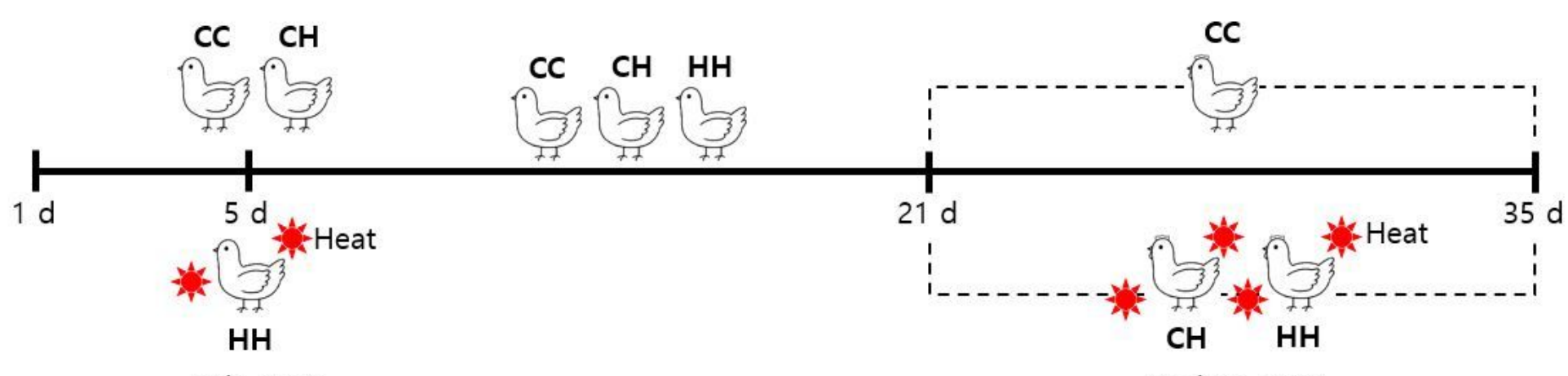

$24 \mathrm{~h}, 40^{\circ} \mathrm{C}$

14 days, $35^{\circ} \mathrm{C}$

\section{Figure 1}

Schematized schedule of heat exposure condition. CC: raised at a suitable temperature without heat exposure; $\mathrm{CH}$ : chronic heat exposed group; $\mathrm{HH}$ : early and chronic heat exposed group. 

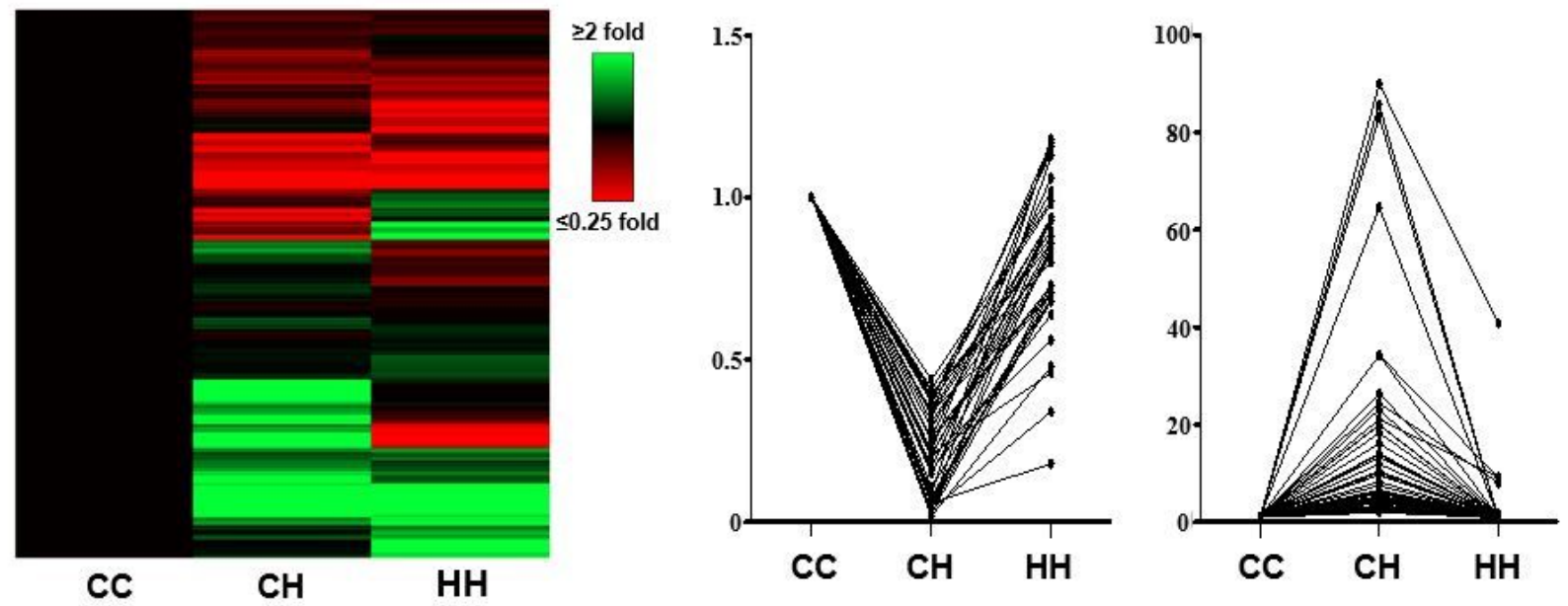

Figure 2

Heat map using differentially expressed proteins and expression patterns of positive effected proteins by early heat. $\mathrm{CC}$ : raised at a suitable temperature without heat exposure; $\mathrm{CH}$ : chronic heat exposed group; $\mathrm{HH}$ : early and chronic heat exposed group. 


\section{a. Biological process}

small molecule metabolic process actin filament-based process

actin cytoskeleton organization

cofactor metabolic process

sulfur compound metabolic process

cellular response to xenobiotic stimulus

cell redox homeostasis

glutathione metabolic process

ether metabolic process

glycerol ether metabolic process
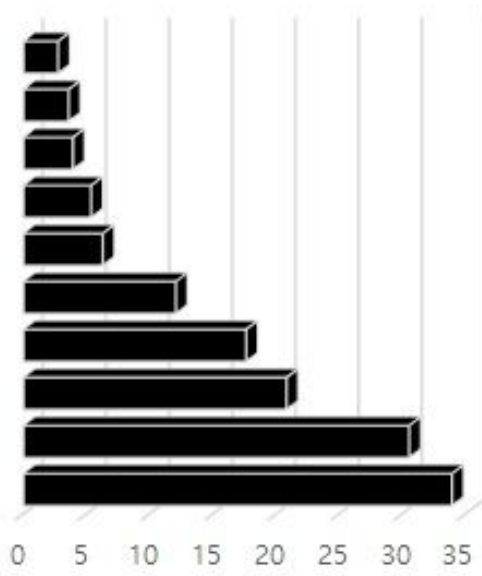

\section{b. Cellular component}

\section{c. Molecular function}

protein-containing complex binding

cytoskeletal protein binding actin binding

ligase activity

actin filament binding

oxidoreductase activity, acting on a sulfur group of donors

disulfide oxidoreductase activity protein disulfide oxidoreductase activity microfilament motor activity peptide disulfide oxidoreductase activity
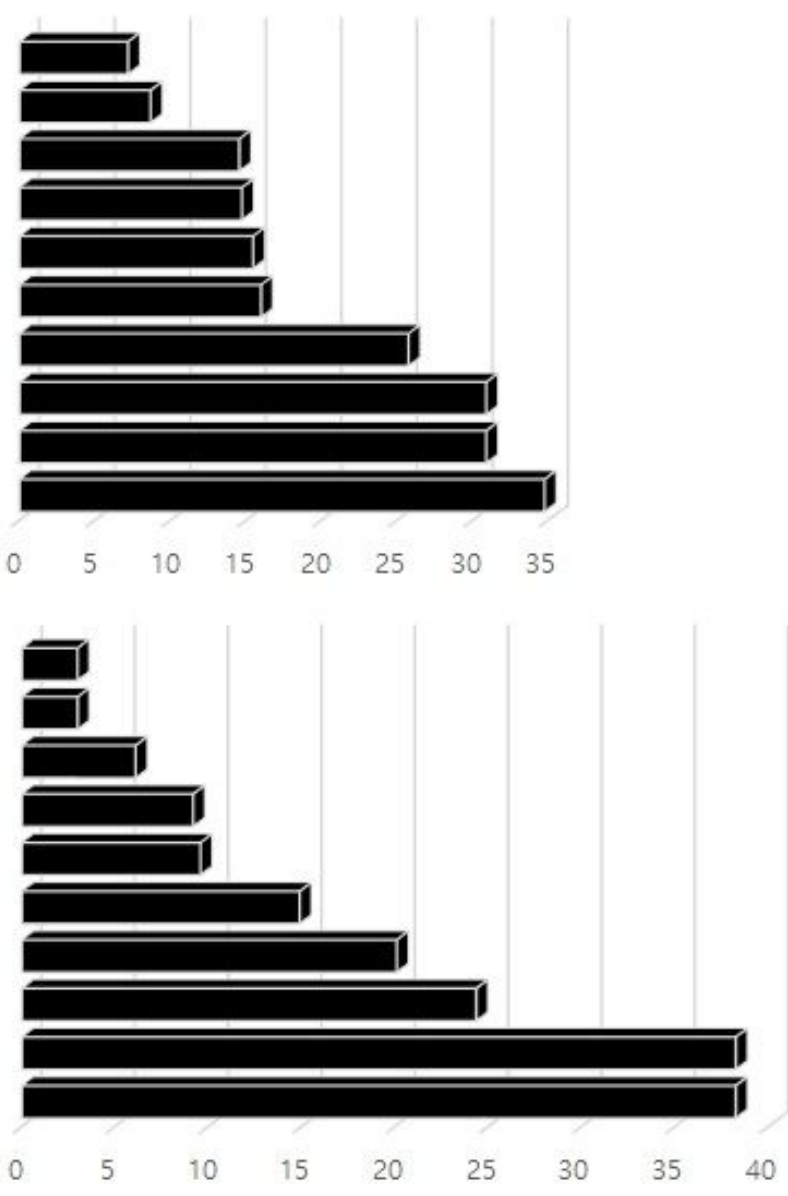

\section{Figure 3}

Gene Ontology terms of positive effected proteins of differentially expressed proteins by early heat exposure 

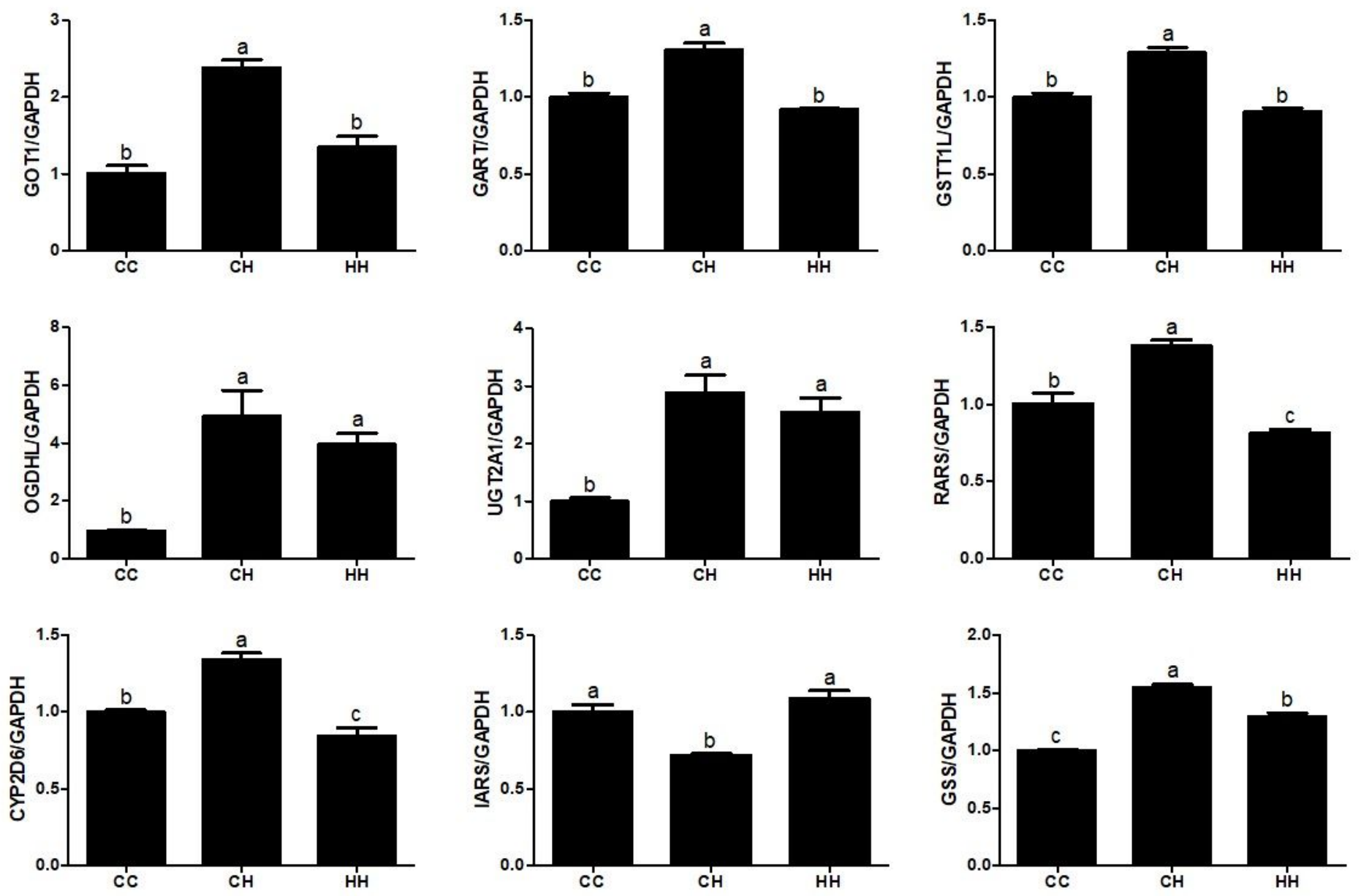

Figure 4

Gene expression in the liver tissue. CC: raised at a suitable temperature without heat exposure; $\mathrm{CH}$ : chronic heat exposed group; $\mathrm{HH}$ : early and chronic heat exposed group. The error bar represent the standard error 


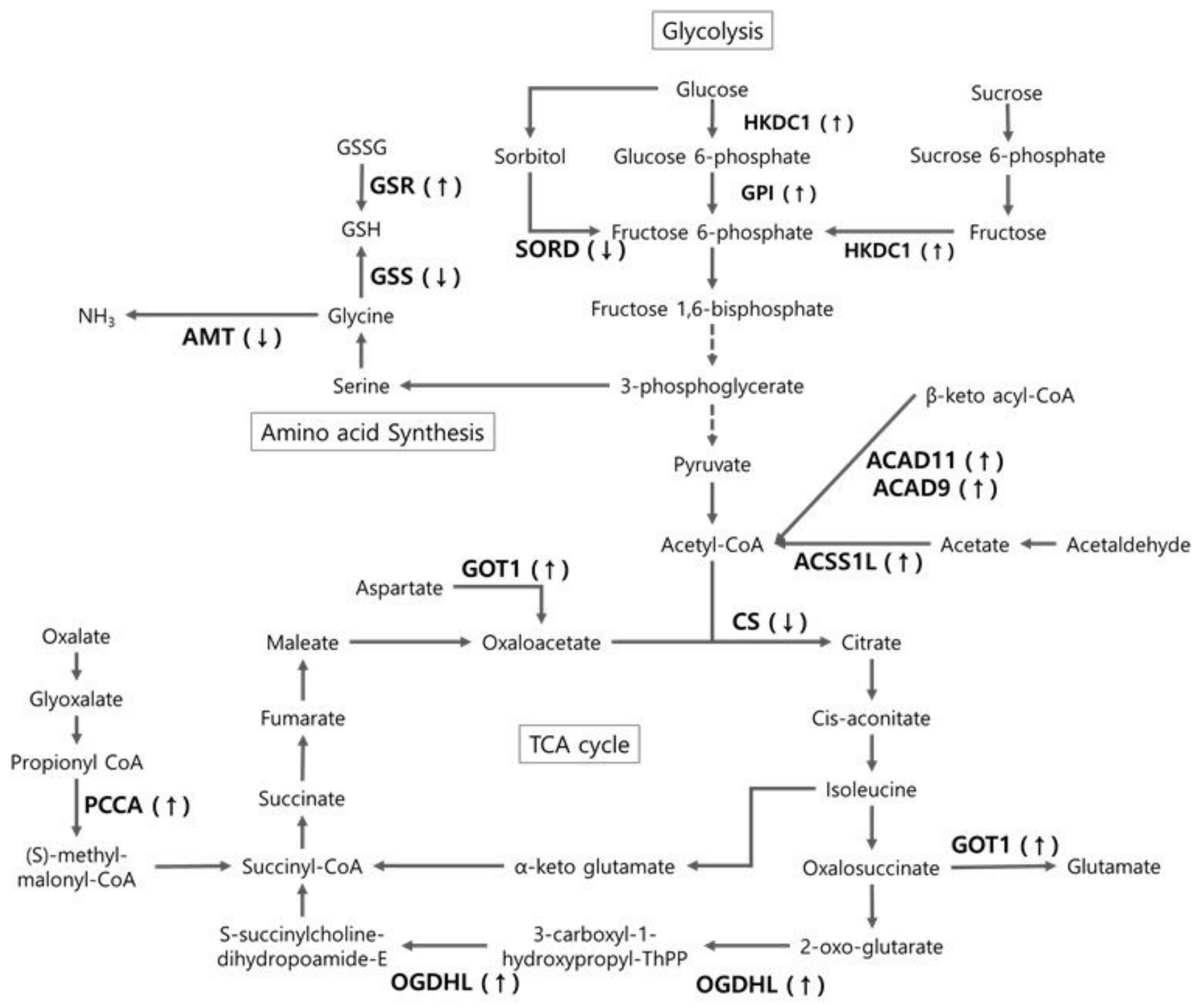

Figure 5

Metabolic schematic of differentially expressed proteins

\section{Supplementary Files}

This is a list of supplementary files associated with this preprint. Click to download.

- renamed22c14.docx

- renamed22c14.docx 